\title{
Les médications dites adjuvantes, générales et locales, dans le traitement de la syphilis. Leur rôle et leur importance.
}

Par le

\author{
Dr. Georges Thibierge, \\ Médecin de l'Hôpital St. Lonis (Paris).
}

Il n'est pas un spécialiste qui se contente de prescrire aux syphilitiques la médication dite spécifique de la syphilis et qui ne sache, à l'occasion et suivant les cas, y ajouter des médications adjuvantes empruntées à la thérapeutique commune et des prescriptions d'hygiène et de diétetique.

L'expérience clinique a, en effet, démontré que, malgré leur efficacité si étonnante et leur action élective sur les lésions syphilitiques, les mercuriaux, les préparations iodiques et iodurées, ${ }^{1}$ ) sont parfois - pour ne pas dire souvent - incapables de produire ฉ̀ eux seuls la guérison complète de tous les accidents syphilitiques, et que, pour guérir un malade dans le minimum de temps et avec le maximum de sécurité, il faut leur associer des médications internes et externes.

Les adjurants thérapeutiques du mercure et de l'iodure de potassium ont pour effet, tantôt de modifier l'état général,

1) L'arséno-benzol a, sur la plupart des lésions syphilitiques ulcéreuses, une action beaucoup plus rapide que le mercure et les iodures. Aussi ne saurait on lui appliquer intégralement la plupart des considérations développées dans ce travail, qui visent les médicatiens mercurielle et iodurée. 
de la mettre en mesure de résister à l'action nocive du virus syphilitique ou de mieux assimiler les mercuriaux et les iodures, tantôt de modifier des lésions locales, en supprimant les infections secondaires, en changeant les conditions de la circulation, au niveau même des syphilides, en y activant les processus de défense des tissus et de l'organisme.

On sait le rôle des maladies antérieures, intoxications ou infections, des viciations générales de la nutrition d'origine héréditaire ou acquise, des divers modes de surmenage dans l'évolution de certains accidents syphilitiques. Les syphilis malignes précoces, si elles sont peut être parfois fonction de la virulence spéciale du contage syphilitique, semblent bien plus sourent encore relever étiologiquement de l'état constitutionnel des sujets: lymphatisme, infections antérieures, misère privations etc. Il est avéré que, dans ces formes morbides, le traitement mercuriel ou le traitement ioduré sont insuffisants à amener la guérison, qu'ils sont souvent même nuisibles: avant de les prescrire, il convient de modifier l'état général du malade, de recourir aux toniques, aux préparations ferrugineuses, aux préparations arsenicales, à la suralimentation, de soumet tre les malades à l'aération continue. Ce traitement, préparatoire pour ainsi dire, met l'organisme en état de recevoir utilement le mercure, assure l'efficacité rapide de celui-ci et la guérison des lésions dans un temps court.

L'efficacité des cures thermales sulfureuses et chlorurées sulfureuses ne tient pas seulement à ce qu'elles permettent, grâce aux propriétés chimiques des eaux, de faire tolérer et absorber une quantité plus considérable de mercure. Elles agissent encore, et je dirai surtout, comme modificateurs de la nutrition générale, comme correcteurs de l'assimilation défectueuse. A ce titre elles sont utiles, au cours de la médication hydrargyrique, chez les sujets surmenés, anémiés par le séjour des villes, entachés de lymphatisme, etc.

Les eaux arsenicales ont, chez les sujets lymphatiques atteints de lésions syphilitiques tenaces, une action souvent aussi arantageuse.

Les cures d'aération continue, les inhalations d'oxygène, la suralimentation jouent, de même, chez les syphilitiques se- 
condaires anémiés par des causes diverses, parfois même chez des syphilitiques tertiaires déprimés par les excès, le surmenage ou les privations, un rôle curatif de premier ordre. J'ai suivi il y a dix ans un malade d'une cinquantaine d'années, atteint de syphilides ulcéreuses serpigineuses de l'avant bras, qui avait subi les traitements mercuriels les plus actifs sans obtenir plus qu'une cicatrisation partielle et passagère. L'usage prolongé de l'iodure de potassium arait entraîné, outre un amaigrissement notable, des troubles digestifs; le malade, vivement impressionné par la longue durée et les récidives incessantes de ses lésions, restait presque continuellement confiné à la chambre t $t$ avait pris en quelques mois l'aspect d'un vieillard, en même temps que ses ulcérations s'étaient étendues, atteignaient la paume de la main et menaçaient de pénétrer jusqu'aux muscles de l'éminence thénar. Des inhalations d'oxygène, le séjour continu dans une pièce aérée par l'ouverture des fenêtres, l'administration des toniques relevèrent rapidement l'état général; les ulcérations ne tardèrent pas à se déterger, à prendre un meilleur aspect, et bientôt la reprise du traitement mercuriel amena dans l'espace de 6 semains la cicatrisation définitive - laquelle s'est toujours maintenue - des syphilides qui lui résistaient depuis plusieurs années.

L'action adjuvante des topiques n'est pas moins importante que celle des médications générales. Elle aussi est bien connue des syphiligraphes.

Elle est mise en œurre, instinctivement pour ainsi dire, dès l'apparition de la syphilis, sur le chancre.

Les topiques, les pansements, les lotions, les pulvérisations sont en réalité des adjuvants de la médication interne, qui, plus que celle-ci, aident à la cicatrisation du chancre, ou mieux combattent les causes qui s'opposent à sa cicatrisation. En supprimant ou en réduisant les infections secondaires, en calmant les réactions inflammatoires qu'elles provoquent, en favorisant la phagocytose et l'épidermisation, caustiques légers, poudres absorbantes, pansements humides, pulvérisations aseptiques réduisent la durée, modérent l'extension du chancre infectant. 
À la période secondaire, les plaques muqueuses, tant à la bouche qu'aux organes génitaux, résistent souvent à la médication mercurielle, tout au moins guérissent lentement lorsqu'elle est seule mise en curre; vient on à pratiquer des attouchements arec le nitrate d'argent, avec le bleu de méthylène, substances dont l'action porte sur les agents d'infection secondaire, on les voit se réparer rapidement. Abandonnées à elles mêmes sans traitement local, alors même que le mercure est administré par les méthodes intensives, les plaques muqueuses hypertrophiques de la vulve persistent de façon désespérante: il suffit de quelques cautérisations soigneusement faites avec le nitrate acide de mercure ou avec un crayon de chlorure de zinc, et de lavages arec des solutions antiseptiques faibles pour en amener la réparation rapide.

Dans les lésions ulcéreuses précoces du tégument, les pansements humides antiseptiques, les attouchements au nitrate d'argent, au nitrate acide de mercure, au chlorure de zinc aident singulièrement l'action du traitement général et régularisent la cicatrisation.

Il en est de même dans les ulcérations tertiaires. En règle générale, on a soin de les panser aseptiquement, tant pour calmer les réactions inflammatoires résultant d'infections surajoutées que pour faciliter leur cicatrisation: les pansements humides, les applications de cataplasmes, les lotions antiseptiques ou simplement aseptiques, les pulvérisations, les applications d'emplâtres n'ont pas d'autre but, aux yeux de la généralité des praticiens, ni d'autre effet, que d'aider à la guérison, laquelle est la conséquence directe de l'action du traitement général. Dans l'opinion courante, tout l'honneur de celle-ci revient au mercure et aux iodures.

Les syphiligraphes ne partagent sans doute pas tous cette opinion simpliste et plus d'un a vu des cas où le prétendu adjurant a joué un rôle capital dans la guérison de lésions syphilitiques. ${ }^{1}$ )

1) N'ayant ni l'intention d'écrire une monographie sur la question traitée ici, ni la prétention de signaler des faits nouveaux, je crois inutile de rapporter les citations, ou les indications bibliographiques des ouvrages et mémoires relatant des faits semblables. Je me contenterai de rappeler 
Pour ma part, il m'est arrivé à plusieurs reprises, pour des motifs divers, de soumettre des sujets atteints de lésions syphilitiques ulcéreuses à des traitements exclusivement locaux et de les voir rapidement guérir d'une façon complète ou à peu près complète.

Tantôt il s'agissait de malades dont l'état général ou quelque altération des voies digestives contreindiquait plus ou moins formellement l'emploi du mercure ou des iodures; en attendant de pouvoir instituer une médication active, je roulais supprimer les causes d'infections secondaires ou les lésions inflammatoires développées au niveau des ulcérations. Tantôt, le diagnostic de syphilis ne s'imposait pas dès le premier jour, une biopsie était nécessaire; un pansement aseptique simple était appliqué pour protéger les surfaces malades et avec l'illusion que. s'il s'agissait de syphilis, il ne modifierait pas l'aspect des lésions; au moment où l'examen histologique était terminé, l'ulcération s'était modifiée et avait perdu tous ses caractères initiaux. Parfois même. les médications mercurielles les plus diverses ajant échoué, il m'était arrivé de les suspendre temporairement avec l'espoir que, après une période de repos thérapeutique, elles auraient plus d'efficacité et je me contentais, pendant cette phase de répit, de modifier les applications locales et de les faire exécuter arec plus de soin, ce la suffisait à amener une amélioration des lésions.

Voici brièvement résumés quelques cas, récemment observés dans mon service, de lésions syphilitiques ulcéreuses, rapidement améliorées sous l'action d'applications externes, sans qu'il ait été fait usage d'aucune préparation mercurielle, iodurée ou arsénicale.

Observation I. Jeanne R., 23 ans, domestique, entre à l'hôpital le 2 septembre 1910 pour une ulcération du tiers supérieur du mollet qui a débuté sans douleurs au mois de juillet, à la suite d'une écorchure.

Actuellement, l'ulcération, ovalaire, du diamètre d'une pièce de 5 francs, a des bords réguliers, non décollés, legèrement saillants, for-

une récente publication de mon excellent collègue et ami B r o c q, à propos d'un fait superposable à ceux que j'étudie: (L. Brocq. Les conditions qui gouvernent la cicatrisation de certaines syphilides ulcéreuses tertiaires. Bulletin médical, 14 Décembre 1910, p. 1141). 
mant un bourrelet violacé et infiltré; le fond est yeu déprimé, irréguiier, de coloration rougeâtre, légèrement suintant; l'ulcération est entourée d'une zone violacée, large de 1 à 2 travers de doigt; sa base est manifestement infiltrée.

La jambe n'est pas variqueuse.

La malade a eu vers 1906 une éruption généralisée, non prurigineuse, et, à la même époque, un ou deux boutons à la vulve, accidents qui ont disparu sans traitement. Elle est sujette aux maxx de tête.

La malade est gardée à l'hôpital pour être traitée par l'arséno. benzol et mise en observation. On se contente de faire sur l'ulcération des lavages à l'eau bouillie et des pansements à la gaze sterilisée.

Au bout de quelques jours, la lésion est remarquablement améliorée: la base est beaucoup moins infiltrée, le suintement a presque complètement cessé; sur son pourtour et à son centre on constate un début d'épidermisation.

On décide alors de différer le traitement par l'arséno-benzol et on continue les pansements comme précedemment.

Le ler octobre, il ne persiste de l'ulcération qu'une cicatrice rose, légèrement infiltrée; mais au niveau des avant-bras est apparue une éruption typique de syphilides circinées, pour laquelle on prescrit un traitement interne.

J'ajouterai que, pendant son séjour à l'hôpital, la malade n'a jamais été astreinte au repos absolu au lit.

Observation II. Jules F., 46 ans, peintre en bâtiments, entre à l'hôpital le 29 octobre 1910 pour des syphilides papulo-tuberculeuses du visage, occupant principalement le pourtour des yeux, la région frontale, la façe dorsale du nez, la joue gauche, les tempes, rappelant une sorte de vaste binocle.

La surface malade est irrégulière, parsemée de profondes dépressions cicatricielles varioliformes, recouverte par places de croûtes jaunes ou brunâtres, mollasses, au dessous desquelles on trouve des ulcérations profondes, mais ne dépassant pas la dimension d'une lentille; par places, surtout sur la joue et sur les tempes, les croûtes sont entourées d'une zone rouge assez étendue.

Le malade a eu la syphilis à l'âge de 25 ans, s'est soigné pendant 3 ou 4 mois, puis a cessé tout traitement.

Les lésions actuelles, qui ont débuté il y a 3 ans au niveau de la racine du nez et n'ont jamais cessé de s'étendre tout on se cicatrisant partiellement, ont été traités par des topiques (emplâtre diachylon, pommade à l'huile de cade); mais le malade n'a pris aucun médicament interne.

Quoique le diagnostic de syphilides papulo-tuberculeuses ne paraisse pas contestable en raison des caractères objectifs, de la continuité des lésions depuis leur début et qu'il soit confirmé par les antécédents du malade et la réaction de Wassermann nettement potitive, je décide 
de faire une biopsie pour répondre à l'objection qui m'est faite qu'il s'agit peut être d'acné nécrotique.

En attendant les résultats de la biopsie, qui devait d'ailleurs confirmer de diagnostic de syphilides (grosse infiltration de cellules rondes et plasmatiques avec quelques rares cellules géantes, disposition nettement périvasculaire des lésions à la partie profonde, intégrité des glandes), je prescris des pulvérisations tièdes d'eau bouillie et des pansements humides.

Dans l'espace de 5 à 6 jours, la rougeur s'atténne, l'infiltration commence à diminuer, quelques croûtes se détachent, et au dessous d'elles la réparation des ulcérations est déjà avancée.

Le 10 novembre, les lésions sont transformées et méconnaissables, on ne peut plus songer à l'acné nécrotique; les croûtes sont tombées presque partout, la rougeur a disparu.

Le 14 novembre, on commence des injections intraveineuses de cyanure de mercure, répétées tous les 2 jours.

Sous l'influence de ce traitement, l'amélioration commencée par le seal traitement local se continue, mais les progrès ne sont pas sensiblement plus rapides que dans la période où on ne faisait que des pulvérisations et des pansements.

Le 4 décembre, il ne reste plus que deux ulcérations très superficielles, une au sourcil gauche, l'autre à la joue. La rougeur a dispara. Les cicatrices tendent déjà à s'aplanir.

Dans les deux observations précédentes, le diagnostic de syphilides ne peut prêter à la contestation.

Dans la suivante, les éléments du diagnostic consistent dans l'aspect objectif des lésions, qui en dehors du chancre simple - avec lequel elles avaient une bien vague ressemblance et que les résultats de l'inoculation permettent d'éliminer - ne rappelaient rien autre que la syphilis et dans l'existence d'antérieure d'une iritis. Je dois reconnaître cependant que la réaction de Wassermann est restée négative.

Observation III. A. J., âgée de 33 ans, n'a d'antre antécédent syphilitique q'une iritis survenue au mois d'avril 1910. A cette époque, elle a reça une série d'injections intraveineuses de cyanure de mercure; depuis le mois de mai, elle n's suivi aucun traitement mercuriel ou ioduré.

Elle entre à l'hôpital le 4 novembje 1910 pour de larges ulcérations, au nombre de 3 , ayant chacune les dimensions d'une pièce de 5 francs, à contour arrondi, à bords légèrement saillants, infiltrés et paraissant un peu décollés, à fond irrégulier, entourées d'une large zone érythémateuse brunâtre, occupant la moitié supérieure de la face antérieure de la jambe gauche; elle porte, en outre, à la face externe de la jambe droite dans sa partie supérieure deux ulcérations de mêmes carac- 
tères et de mêmes dimensions que celles de la jambe gauche. Ces lésions datent de la fin de septembre.

Jusqu'à son entrée à l'hôpital, la malade a fait des pamements humides et des lavages à l'eau oxygénée.

Lors de son entrée, le diagnostic étant quelque peu ambigu avec le chancre simple, on fait une inoculation et on se contente de prescrire le repos au lit et des pansements à l'eau bouillie.

Les jours suivants, les ulcérations se détergent, les bords s'affaissent; au bout d'une quinzaine de jours, elles sont en voie de réparation manifeste, leurs dimensions se sont réduites de près de moitié.

Le 23 novembre, la réparation s'est accusée; on remplace les pansements bumides par des attouchements à la teinture d'iode et des pansements secs; la malade est autorisée à se lever et à marcher modérément.

Le ler décembre, on note l'état suivant: L'une des ulcérations de la jambe droite est complètement fermée, remplacée par une cicatrice rosée, recouverte de squames larges et minces; l'sutre n'est plus représentée que par une surface rouge, légèrement bourgeonnante, de la largeur d'une pièce de 50 centimes, entourée d'une cicatrice. A la jambe gauche, les ulcérations sont réduites à des surfaces variant de la dimension d'une pièce de 50 centimes à celle d'une pièce de 20 centimes, peu profondes, à fond rouge et légèrement bourgeonnant, à bords nets, non décollés, sans infiltration de leur base, donnant issue à une petite quantité de sérosité. Les parties adjacentes à ces petites ulcérations sont représentées par une cicatrice rosée, recouverte par endroits de squames larges peu adhérentes ou de petites croûtes brunâtres qui s'enlèvent facilement et au dessous desquelles l'épiderme est entièrement reconstitué; ces squames sont dûes surtout aux applications de tincture d'iode. négative.

L'inoculation faite lors de l'entrée de la malade est restée

La réaction de Wassermann a également, par contre, été negative.

Le fait suivant est plus remarquable encore. Il s'agit d'une malade atteinte de syphilides malignes précoces ayant, sans interruptions récidivé pendant plus de trois ans: les préparations les plus variées de mercure, d'iodure, d'arsenic, des topiques également divers avaient été prescrits et supportés par la malade sans jamais arrêter définitivement le cours de ces ulcérations, déterminant la cicatrisation de quelques unes d'eatre elles, cicatrisation généralement suivie à court intervalle de la reproduction des lésions ulcéreuses. Ayant, dans diverses affections ulcéreuses, obtenu des résultats remarquables par l'emploi de badigeonnages de coaltar suivant la méthode de Dind (Lausanne), je supprimai toute médication interne et je fis faire sur quelques 
ulcérations des badigeonnages de coaltar: au bout de quelques jours, ces ulcérations étaient cicatrisées ; successivement, la même médication fut appliquée aux diverses ulcérations et successivement elles se cicatrisèrent toutes dans l'espace de peu de jours. Et la malade qui était restée trois ans passés dans mon service sans avoir plus de quelques jours de répit, est actuellement guérie de toutes ses ulcérations depuis plus de six mois, bien qu'elle n'aie plus suivi aucun traitement général depuis les premières applications de coaltar.

Voici, arec plus de détails, cette observation remarquable que j'ai communiquée en juillet dernier à la Société française de dermatologie.

Observation IV. B. est entrée le 9 octobre 1906 dans mon service. Elle était alors âgée de 16 ans et atteinte de syphilides malignes précoces ulcéreuses occupant les membres et la face; fatiguée par des privations multiples, n'ayant pas d'appétit, elle était très amaigrie.

Traitée par la suralimentation, les inhalations d'oxygène, l'iodure de fer, elle s'amóliora; les lésions ulcéreuses, sous l'influence de cautérisations au nitrate acide de mercure, puis d'injections mercurielles, s'amendèrent, se cicatrisèrent partiellement, puis ne tardèrent pas à se reproduire.

Pendant les annés 1907, 1908 et 1909, les alternatives d'amélioration, avec cicatrisation partielle et d'aggravation, de reprises du processus ulcéreux se succédèrent, les améliorations suivant l'emploi des divers composés mercuriels (calomel, injections intraveineuses de cyanure de mercure, injections d'huile grise, injections d'huile biiodurée etc.) ou des iodures et les applications de topiques varies, aseptiques on caustiques, mais ne se prolongeant guère, quelque soit le médicament ou le topique employé, pendant plus de 15 à 20 jours. Les ulcérations se reproduisaient au niveau de la cicatrice des lésions anciennes ou en des points jusque là indemnes. Des lésions nlcéreuses se produisirent au front, au visage, détruisant près de la moitié des narines, au voile du palais, provoquant la chute de la luette et d'une partie des piliers, aux nembres, -où plusieurs, atteignant jusqu'à 10 centimètres de large, mettaient à nu les aponévroses et les muscles, eutravant les mouvements de l'épaule et du coude droit; d'autres moing étendues occupaient les membres.

Au mois d'avril dernier, une partie des ulcérations étaient cicatricées, notamment celles du nez, du voile du palais; mais il restait encore de très larges ulcérations an niveau du front, le la partie externe de l'épaule droite, à la face postérieure des avant-bras, à la face interne des jambes, ulcérations à bords infiltrés, à fond irrégulier, suppurant abondamment, douloureuses à la pression, celles du front atteignant jusqu'à l'os frontal. 
C'est alors que je fis faire sur une partie des ulcérations des badigeonnages au coaltar, suivant la méthode de $\mathrm{Dind}$, tout en supprimant systématiquement toute médication générale, aussi bien tonique que mercurielle. Au bout de 6 à 7 jours, les ulcérations ainsi traiţ́es commencèrent à se cicatriser de la périphérie vers le centre; en même temps, les sécretions se tarissaient et, dans l'espace de 20 à 25 jours, la cicatrisation était complète. Successivement toutes les ulcérations persistantes furent traitées de la même façon et se réparèrent avec la même rapidité. L'ulcération de l'épaule, la plus profonde de tontes, mit un pen plus longtemps à se réparer. Les ulcérations des jambes, conservées les dernières comme témoins pour bien etablir la rôle des badigeon. nages de coaltar, n'avaient, avant d'y être soumises, présenté aucune modification.

Le 25 mai, exactement 6 semaines après le commencement des badigeonnages au coaltar, les ulcérations étaient toutes réparées; les cicatrices des ulcérations de l'épaule droite et des avant-bras formaient des bandes fibreuses gênant les mouvenents des articulations correspondantes.

L'état général s'était amélioré, l'appétit était devenu neilleur et le sommeil était bon.

La malade fut envoyée à la campagne, d'où elle revint au bout de $\mathbf{B}$ semaines, complètement guérie et ayant sensiblement engraissé.

Elle est restée pendant tout l'été sans aucun traitement.

Le 28 novembre, elle est revenue nous voir. Les ulcérations ne se sont pas reproduites. Les brides cicatricielles de l'épaule ot des coudes se sont assouplies. L'atrophie musculaire des membres, assez prononcée au mois de mai, a complètement disparu. Les forces sont revenues, la malade peut se livrer à un travail assez fatigant. Elle a notablement engraissé et, sauf les cicatrices, ne présente plus aucune trace de sa syphilis.

Elle va, pour la première fois depuis le mois d'avril, être soumise de nouveau au traitement mercuriel.

Cette demière observation ne peut être comparée absolument aux précédentes. La résistance des ulcérations à toutes les médicatious syphilitiques internes, comme les reprises incessantes des lésions au cours même de ces médications, comme aussi l'absence de toute récidive depuis que la malade guérie par les applications de goudron a cessé tout traitement, pourrait laisser supposer que les ulcérations araient perdu toute qualité syphilitique, qu'jl s'agissait exclusivement de lésions banales ou d'origine microbienne externe aidées seulement par Je terrain et l'état général défectueux de la patiente.

Il n'en est pas moins vrai que ces ulcérations avaient toujours conservé les caractères morphologiques des syphilides 
malignes précoces et que, au moment où le traitement local a été institué, il était impossible de porter un autre diagnostic.

L'observation méritait donc bien, malgré tout ce qu'elle a d'extraordinaire, d'invraisemblable pourrait on dire, de figurer ici.

Les faits que je viens de rapporter montrent que, à l'ex clusion de tout traitement antisyphilitique, des pansements locaux peuvent amener la cicatrisation d'ulcérations manifestement syphilitiques.

Il est nécessaire, je crois, de préciser: ulcérations syphilitiques, et non: lésions syphilitiques quelconques; je ne sache pas, en effet, qu'on obtienne des résultats pareils dans des cas de lésions syphilitiques non ouvertes, papuleuses ou papulotuberculeuses.

$\grave{A}$ aucun titre, je ne voudrais en conclure ou en inférer que le traitement antisyphilitique général doive être remplacé par des pansements locaux.

Malgré tout l'intérêt des faits précédents, je reste convaincu que le traitement général doit être prescrit, en même temps que le traitement local, chez les sujets porteurs de lésions syphilitiques ulcéreuses, et je tiens à déclarer qu'il doit être prescrit aux sujets qui auraient été guéris de lésions syphilitiques ulcéreuses par des applications exclusivement locales. Je suis, pour ma part, trop assuré de la réalité de ce fait que, après la guérison apparente ou guérison macroscopique des lésions syphilitiques, il persiste des altérations histologiques, et que celles ci ne sont stérilisées et rendues inaptes à récidiver que par une médication générale suffisamment prolongée.

Chez la dernière des malades dont j'ai rapporté l'observation, je suis resté pendant six mois sans prescrire de traitement interne; je ne suis resté dans l'abstention thérapeutique que parcequ'il s'agissait d'un cas exceptionnel et que je voulais donner la preuve formelle de l'efficacité de la cure locale; j'y étais encouragé d'ailleurs par ce fait que, pendant plus de 3 ans, la malade avait été pour ainsi dire saturée de mercure. Dès maintenant, je reprends chez elle la cure hydrargyrique.

Il est deux conclusions à tirer des faits précedents. 
La première et la plus importante est que le traitement local des ulcérations syphilitiques n'est pas seulement un adjuvant, plus ou moins accessoire, de la médication interne, adjuvant qui peut être plus ou moins impunément négligé, mais bien au contraire qu'il représente un élément important de la guérison.

D'où cette déduction que, lorsqu'on veut étudier la valeur curative d'une méthode de traitement de la syphilis, il faut ou bien ne lui associer aucun traitement local des ulcérations, ce traitement fût-il uniquement un pansement humide aseptique, ou bien faire entrer dans l'appréciation des résultats. obtenus l'intervention de ce pansement.

La seconde conclusion est d'ordre diagnostique.

En matière de syphilis, plus qu'en toute autre matière, nous sommes toujours enclins à invoquer le vieil adage: Naturam morborum ostendunt curationes, quoiqu'il soit, en pareille matière, de plus en plus contestable.

Les observations du genre de celles que je viens de rapporter donnent un nouveau motif de méfiance à son égard: elles s'opposent à ce qu'on nie la nature syphilitique d'une ulcération par ce seul motif qu'elle a guéri, fut-ce complètement et rapidement, sous l'influence d'un simple traitement local. On devra done, toutes les fois où un diagnostic paraîtra douteux et où les circonstances ne permettront pas de l'éclairer par la biopsie, s'abstenir de tout traitement local, si on veut pouvoir ultérieurement faire état au point de vue du diagnostic des résultats soit de l'expectation soit d'une médication interne. 